\title{
Applications of the Transport Integrals in Solid-State Physics and in Electron Spin Relaxation
}

\author{
T. RadczYK, S.K. HofFMAnN* And J. Goslar \\ Institute of Molecular Physics, Polish Academy of Sciences \\ Smoluchowskiego 17, 60-179 Poznań, Poland
}

(Received September 12, 2003)

\begin{abstract}
Transport integrals $I_{n}\left(\Theta_{\mathrm{D}} / T\right)$ are reviewed with their applications in solid-state physics, molecular dynamics, and electron spin-lattice relaxation. Analytical approximations of $I_{n}$ for $n=2-8$ are proposed as applicable for computer fitting procedures in the range of the variable $x=\Theta_{\mathrm{D}} / T$ from 0.1 to 40 . The results are applied for description of the spin-lattice relaxation data collected for $\mathrm{Cu}^{2+}$ ions in triglycine sulphate and are compared with relaxation data for $\mathrm{Cu}^{2+}$ and $\mathrm{Mn}^{2+}$ in $\left(\mathrm{NH}_{4}\right)_{2} \mathrm{Mg}\left(\mathrm{SO}_{4}\right)_{2} \cdot 6 \mathrm{H}_{2} \mathrm{O}$.
\end{abstract}

PACS numbers: $63.90 .+\mathrm{t}, 76.30 .-\mathrm{v}$

\section{Introduction}

Many physical properties of crystalline and amorphous solids are associated with crystal lattice vibrations (phonons). Phonons play a key role in bulk physical properties such as specific heat, thermoelectric phenomena, electrical and thermal conductivity and other transport properties of solids. Theoretical phonon spectra in the first approximation are usually considered within the Debye model which for acoustic phonons assumes the density of phonon states $g(\omega) \propto \omega^{2}$ with the cut-off frequency $\omega_{\mathrm{D}}=k \Theta_{\mathrm{D}} / \hbar$, where $\Theta_{\mathrm{D}}$ is the Debye temperature.

When the whole phonon spectrum is involved in a physical phenomenon, then theoretical expressions contain, as a rule, a term

$$
I_{n}\left(\Theta_{\mathrm{D}} / T\right) \equiv \int_{0}^{\Theta_{\mathrm{D}} / T} \frac{x^{n} \mathrm{e}^{x}}{\left(\mathrm{e}^{x}-1\right)^{2}} \mathrm{~d} x,
$$

which is called the transport integral of the $n$-th order. The values of the transport integrals $I_{n}(x)$ are not calculable analytically and their values are tabulated in

*corresponding author; e-mail: skh@ifmpan.poznan.pl 
various ranges of the variable $x[1-4]$ and/or can be found using nowadays computer algebra programs. However, analytical approximations of $I_{n}(x)$ are needed for using in computer routines for an effective fit or simulations of temperature variations of various physical parameters. We have already published such approximation for integral $I_{8}$, applied to an analysis of electron spin-lattice relaxation data of $\mathrm{Cu}^{2+}$ ions $[5,6]$.

In this paper we describe situations where various $I_{n}$ appear in solid-state physics, in molecular dynamics and in electron spin-lattice relaxation. We present analytical approximations for $I_{2}-I_{8}$ in the range of $x$-values from 0.1 to 40 . An application of the obtained expressions is presented for spin-lattice relaxation data of $\mathrm{Cu}^{2+}$ and $\mathrm{Mn}^{2+}$ in a few single crystals.

\section{Transport integrals in solid-state physics}

An origin of the transport integral can be well explained considering the heat capacity of a solid $[7,8]$. The internal energy $U$ of a solid resulting from thermal lattice vibrations can be calculated as

$$
U=\int_{0}^{\omega_{\mathrm{D}}} \hbar \omega \frac{g(\omega)}{\exp (\hbar \omega / k T)-1} \mathrm{~d} \omega,
$$

where all phonon modes of energy $\hbar \omega$ are summarized for a continuous phonon spectrum with density of states $g(\omega)$ assuming the Bose-Einstein statistics for phonons with phonon occupation number $[\exp (\hbar \omega / k T)-1]^{-1}$. For $g(\omega) \propto \omega^{2}$ and $x=\hbar \omega / k T$ one can write

$$
U \propto T^{4} \int_{0}^{\Theta_{\mathrm{D}} / T} \frac{x^{3}}{\mathrm{e}^{x}-1} \mathrm{~d} x .
$$

Specific heat is then

$$
C_{V}=\frac{\partial U}{\partial T} \propto T^{3} \int_{0}^{\Theta_{\mathrm{D}} / T} \frac{x^{4} \mathrm{e}^{x}}{\left(\mathrm{e}^{x}-1\right)^{2}} \mathrm{~d} x=T^{3} I_{4}\left(\Theta_{\mathrm{D}} / T\right) .
$$

Transport integral $I_{4}$ appears also in theory of thermal conductivity of solids $[8,9]$. Integral $I_{5}$ occurs in description of electrical conductivity [8], and various order transport integrals were used in thermoelectric force theory [10]. Transport integral $I_{2}$ is used in calculations of the electronic contribution to the specific heat of metals, where integration is performed not over the phonon spectrum but over the electron states up to the Fermi level $E_{\mathrm{F}} / k T[7]$.

\section{Transport integrals in molecular dynamics}

Transport integrals occur in microscopic physics when one considers reorientations of molecules or molecular groups between potential minima separated by energy barriers. The reorientations are induced by phonons and, besides the 
classical over-barrier jumps, various mechanisms of barrier crossing have been considered [11-13]. The entire phonon spectrum is involved when two-phonon Raman processes induce reorientations. The expressions containing $T^{n-1} I_{n}\left(\Theta_{\mathrm{D}} / T\right)$ then commonly appear.

For a system with molecules localized in a few equivalent potential wells at low temperatures the quantum mechanical tunnelling mixes the ground-state wave functions. When temperature increases, the phonons modulate potential surface producing time dependent changes in the localization energy, i.e. producing dynamical inequivalence between potential minima. As a result the reorientation rate due to the two-phonon Raman processes becomes temperature dependent and proportional to $\Gamma^{2} T^{3} I_{2}\left(\Theta_{\mathrm{D}} / T\right)$; here $\Gamma$ is a tunnelling splitting [11].

Another temperature dependence of the reorientation rate occurs when the phonon-assisted (phonon-induced) tunnelling mechanism operates, i.e. when a tunnelling via oscillating potential barrier exists. Oscillations of the barrier height or distance between potential minima (barrier width) allow temperature depending tunnelling even between inequivalent potential wells. When the two-phonon Raman processes produce these oscillations, the reorientation rate is proportional to $T^{7} I_{6}\left(\Theta_{\mathrm{D}} / T\right)[12]$.

Reorientations can appear also between potential minima produced by the Jahn-Teller effect when wave functions of the vibronic states in neighbouring wells are mixed. Phonon-induced mechanism via two-phonon Raman processes leads in such a case to temperature dependence of the reorientation rate proportional to $T^{5} I_{4}\left(\Theta_{\mathrm{D}} / T\right)$ [13], when thermal energy is larger than the inequivalence between the Jahn-Teller distorted configurations.

\section{Transport integrals in electron spin-lattice relaxation}

Two-phonon Raman processes dominate electron spin-lattice relaxation, i.e. a thermalization of a spin system in crystalline solids, at intermediate and high temperatures. The Raman process is a scattering of lattice phonons on electron spins with simultaneous change of phonon energy and the spin flip between the Zeeman levels.

Probability of the relaxation transition between spin states $a$ and $b$ can be calculated in the first-order time-dependent perturbation theory with a second-order perturbation by the phonon-induced strains $\varepsilon_{I}$, i.e. taking the third term in the crystalline potential expansion: $V=V_{0}+\sum_{n} V^{\prime} \varepsilon_{n}+\sum_{n, m} V^{\prime \prime} \varepsilon_{m} \varepsilon_{n} \ldots[14,15]$. This approach leads to the spin-lattice relaxation rate $1 / T_{1}$ in the form

$$
\frac{1}{T_{1}}=\left\langle a\left|V^{\prime \prime}\right| b\right\rangle^{2} \frac{9}{4 \pi^{3} \rho^{2} v^{10}}\left(\frac{k T}{\hbar}\right)^{7} I_{6}\left(\Theta_{\mathrm{D}} / T\right),
$$

where $\rho$ is a crystal density and $v$ is the sound velocity in the crystal. 
When a higher singlet spin state $c$ exists with energy $\Delta$ larger than the continuum phonon energies, which is common for iron-group transition ions with even number of unpaired electrons, the contribution to the relaxation can appear via the state $c$. This state is already admixed to the ground state spin doublet thus the transitions to the state are described as virtual. The contribution can be treated to the first order in the lattice strains using the second-order perturbation theory, and the resulting relaxation rate is $[14,15]$ :

$$
\frac{1}{T_{1}}=\frac{\left\langle a\left|V^{\prime}\right| c\right\rangle^{2}\left\langle c\left|V^{\prime}\right| b\right\rangle^{2}}{\Delta^{2}} \frac{9}{4 \pi^{3} \rho^{2} v^{10}}\left(\frac{k T}{\hbar}\right)^{7} I_{6}\left(\Theta_{\mathrm{D}} / T\right) .
$$

Equations (5) and (6) describe the relaxation of the non-Kramers ions.

For the Kramers ions having odd number of unpaired electrons there exists at least a single-excited spin doublet $c$ and $d$ with energy $\Delta$ higher than the Debye energy $k \Theta_{\mathrm{D}}$. In such a case the effective relaxation rate is

$$
\frac{1}{T_{1}}=\frac{\left\langle a\left|V^{\prime}\right| c\right\rangle^{2}\left\langle c\left|V^{\prime}\right| b\right\rangle^{2}}{\Delta^{4}} \frac{9}{\pi^{3} \rho^{2} v^{10}}\left(\frac{k T}{\hbar}\right)^{9} I_{8}\left(\Theta_{\mathrm{D}} / T\right) .
$$

For high-spin ions $(S>1 / 2)$ like $\mathrm{Mn}^{2+}$, the relaxation can take place within the spin multiplet manifold of the fine structure. In such a case the relaxation rate is $[16]$ :

$$
\frac{1}{T_{1}}=\frac{c \hbar^{2}}{\rho^{2} \Delta^{4} v^{10}}(k T)^{5} I_{4}\left(\Theta_{\mathrm{D}} / T\right) .
$$

Reorientations of a paramagnetic centre can also produce electron spin relaxation when they are accompanied by spin flips. In dynamic Jahn-Teller effect the reorientations between distorted configurations produce spin flips due to an anisotropy in EPR $g$-factor and hyperfine coupling, with the spin-lattice relaxation time $T_{1} \propto \tau$, where $\tau$ is a correlation time of the reorientation [13]. Thus, one can expect $1 / T_{1} \propto T^{3} I_{2}\left(\Theta_{\mathrm{D}} / T\right)$ or $1 / T_{1} \propto T^{7} I_{6}\left(\Theta_{\mathrm{D}} / T\right)$ depending on the reorientation mechanism as described in the previous subsection. Except for these mechanisms a direct coupling via phonons between the states of opposite spins in neighbouring potential wells can exist as a consequence of the spin-orbit coupling to the excited states [17]. In such a phonon-assisted spin-orbit induced tunnelling the two-phonon Raman process produces relaxation rate proportional to $T^{7} I_{6}\left(\Theta_{\mathrm{D}} / T\right)[18,19]$.

\section{Transport integral values}

Transport integrals of any order cannot be calculated analytically in the whole range of $x$-parameter. However, at low temperatures and also at temperatures higher than the Debye temperature $\Theta_{\mathrm{D}}$ the $I_{n}$-values can be well evaluated $[1,20]$. For low temperatures, where $x \rightarrow \infty$, i.e. when $T \ll \Theta_{\mathrm{D}}$ : 


$$
I_{n} \cong n !,
$$

which for spin relaxation measurements leads to the $T^{n+1}$ temperature dependence of the relaxation rate. At high temperatures $T>\Theta_{\mathrm{D}}$ :

$$
I_{n}=\frac{x^{n-1}}{n-1}
$$

and the spin-lattice relaxation rate displays quadratic temperature dependence $1 / T_{1} \propto T^{2}$ for all $n$-index values.

In intermediate temperature range numerical calculations can be performed expanding $I_{n}$ in a power or the Bernoulli series $[1,20]$. The most comprehensive tables for $n=2-17$ and $x=\Theta_{\mathrm{D}} / T$ up to 25 or 40 are published in paper [1].

We have found that $I_{n}(x)$ can be well approximated analytically by a power series or/and exponential function $I_{n}(x)=\exp (s)$ where $s=\sum_{i=0}^{n} A_{i}(\ln x)^{i}$ depending on the range of $x$. The approximations with parameters are listed in Table I for $x=0.1-40$ and plots of $I_{4}(x)$ and $I_{8}(x)$ are shown in Fig. 1. The plots show practically the temperature behaviour of the transport integrals below the Debye temperature, which is quasi-linear for intermediate $x$ values and becomes temperature independent for high $x$ values with $I_{n}(x)=n$ ! The Debye temperature of various solids varies in the range $\Theta_{\mathrm{D}}=40-1000 \mathrm{~K}$, whereas experiments are usually performed in the temperature range $4-400 \mathrm{~K}$. Thus, the limits of the $x$-parameter are $x_{\min }=0.004$ and $x_{\max }=10$. Value for $x<0.1$, which are be-

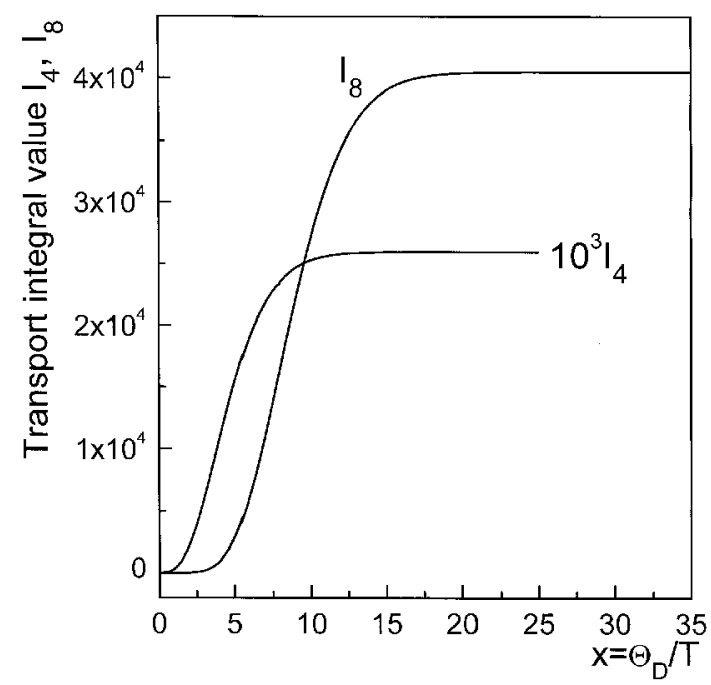

Fig. 1. Transport integral $I_{4}$ (gained $10^{3}$ times) and $I_{8}$ dependence on the variable $x=\Theta_{\mathrm{D}} / T$ plotted from analytical approximations showed in Table I. The high $x$-value limits are equal to $4 ! \times 10^{3}$ and 8 ! for $I_{4}$ and $I_{8}$, respectively. 
TABLE I

Analytical approximations of the transport integrals $I_{2}-I_{8}$ for $x=\Theta_{\mathrm{D}} / T$ in the range 0.1 to 40 .

\begin{tabular}{|c|c|c|c|c|c|c|c|}
\hline \multicolumn{8}{|c|}{$\begin{array}{l}\text { For } n=3-8: I_{n}=\exp \left[A_{0}+A_{1} \ln x+A_{2}(\ln x)^{2}+\ldots A_{k}(\ln x)^{k}\right] \text {; } \\
\text { for } n=2: I_{2}=A_{0}+A_{1} x+A_{2} x^{2}\end{array}$} \\
\hline Integral & $I_{2}$ & $I_{3}$ & $I_{4}$ & $I_{5}$ & $I_{6}$ & $I_{7}$ & $I_{8}$ \\
\hline Range & $0.1-2.1$ & $0.1-25$ & $0.1-11$ & $0.1-10$ & $0.1-18.6$ & $0.1-10$ & $0.1-16.6$ \\
\hline$A_{0}$ & -0.02491 & -0.7376 & -1.14891 & -1.44114 & -1.66685 & -1.85367 & -2.00976 \\
\hline$A_{1}$ & 1.09979 & 1.91425 & 2.89792 & 3.89012 & 4.86552 & 5.87686 & 6.87231 \\
\hline$A_{2}$ & -0.09483 & -0.05577 & -0.08817 & -0.10866 & -0.12413 & -0.12136 & -0.12936 \\
\hline$A_{3}$ & - & -0.03968 & -0.05101 & -0.0648 & -0.03392 & -0.07655 & -0.0788 \\
\hline$A_{4}$ & - & 0.04893 & -0.03816 & -0.02906 & -0.02587 & -0.03519 & -0.03222 \\
\hline$A_{5}$ & - & -0.01342 & -0.01573 & -0.01472 & -0.03595 & -0.01385 & -0.01593 \\
\hline$A_{6}$ & - & -0.00808 & 0.00198 & -0.00362 & -0.00527 & -0.00359 & -0.00549 \\
\hline$A_{7} \times 10^{4}$ & - & 16.9 & 17.2 & 15 & 53.9 & 3.7522 & 9.85532 \\
\hline$A_{8} \times 10^{4}$ & - & -5.80788 & - & 5.73554 & 6.40378 & 4.57258 & 6.21152 \\
\hline$A_{9} \times 10^{5}$ & - & - & - & -2.7857 & -28.518 & 6.47625 & -1.857 \\
\hline \multicolumn{8}{|c|}{$I_{n}=y_{0}+a_{1} \exp \left[-\left(x-x_{0}\right) / t_{1}\right]$} \\
\hline Integral & $I_{2}$ & $I_{3}$ & $I_{4}$ & $I_{5}$ & $I_{6}$ & $I_{7}$ & $I_{8}$ \\
\hline Range & $2.1-25$ & - & $11-25$ & $10-25$ & $18.6-30$ & $10-30$ & $16.6-40$ \\
\hline$y_{0}$ & 3.2899 & - & 25.97599 & 124.45277 & 732.48735 & 5086.87142 & 40486.52036 \\
\hline$a_{1}$ & -1.54588 & - & -0.18383 & -8.18877 & -0.18479 & -1148.10757 & -1534.32091 \\
\hline$x_{0}$ & 2 & - & 12 & 10 & 20 & 10 & 15 \\
\hline$t_{1}$ & 1.67862 & - & 1.38735 & 1.65099 & 1.37037 & 2.10274 & 1.79496 \\
\hline
\end{tabular}

yond the range of our analytical approximations, can be calculated as for the low temperature limit of $I_{n}(x)$ as $I_{n}(x)=x^{n-1} /(n-1)$.

\section{Applications}

We have applied the analytical approximations of the $I_{n}(x)$ integrals in a computer fit functions describing electron spin-lattice relaxation data of iron-group transition ions in single crystals. The temperature dependence of the relaxation rate $1 / T_{1}$ was parameterised as

$$
\frac{1}{T_{1}}=a T+b T^{n+1} I_{n}\left(\Theta_{\mathrm{D}} / T\right),
$$

where $n=8$ for $\mathrm{Cu}^{2+}$ ion and $n=6$ for $\mathrm{Mn}^{2+}$ ion according to Eqs. (6) and (7). The fitting functions were written in LabTalk (similar to C) language of ORIGIN software. For the function with $I_{8}$ the routine, with a direct process contribution $(a * x)$ and $a, b$, and $d=\Theta_{\mathrm{D}}$ as parameters, has the form 


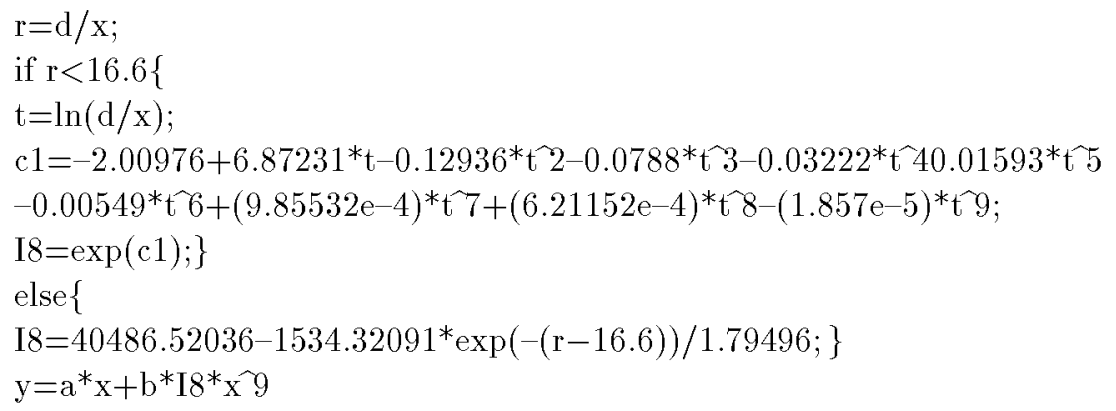

The fitting procedure was applied to experimental data of $\mathrm{Cu}^{2+}$ and $\mathrm{Mn}^{2+}$ in the Tutton salt crystals $\left(\mathrm{NH}_{4}\right)_{2} \mathrm{Mg}\left(\mathrm{SO}_{4}\right)_{2} \cdot 6 \mathrm{H}_{2} \mathrm{O}$ which we already have published $[21,22]$. The fitting parameters $a, b$, and $\Theta_{\mathrm{D}}$ are summarised in Table II. Moreover, we have made electron spin-lattice relaxation measurements by electron spin echo (ESE) technique for $\mathrm{Cu}^{2+}$ ions in triglycine sulphate (TGS) single crystals. The measurements were performed on a BRUKER ESP380E FT/CW spectrometer in temperature range $4-120 \mathrm{~K}$ where ESE signal was detectable. The spin-lattice relaxation time $T_{1}$ was measured by saturation recovery method using a $48 \mathrm{~ns}$ saturating pulse, which excited single hyperfine line $\left(m_{I}=-1 / 2\right)$ in parallel orientation of the $\mathrm{Cu}$ (glycine $)_{2}$ complexes similarly to the triglycine selenate (TGSe) measurements [5]. Two $48 \mathrm{~ns}$ pulses with a 120 ns interpulse interval induced the ESE signal, and no effects from spectral and instantaneous diffusion were observed. The results of measurements are shown as black circles in Fig. 2, where they are

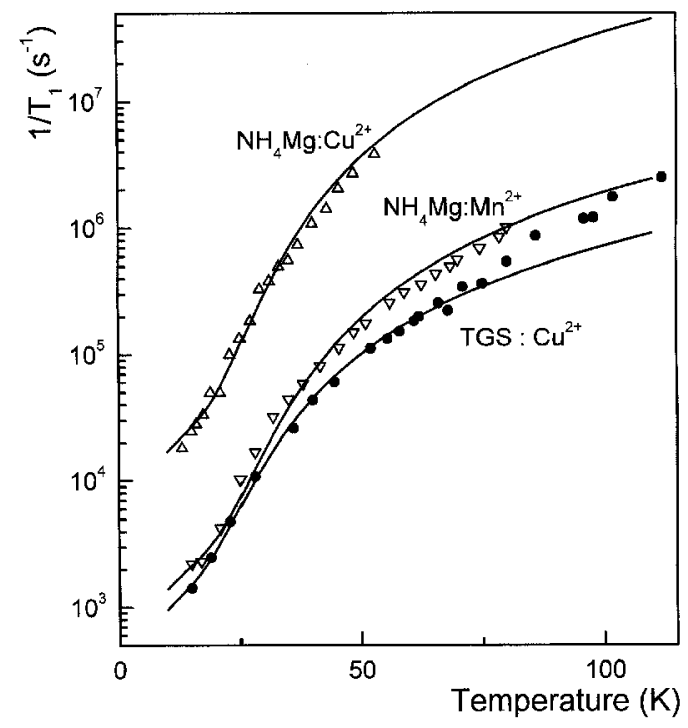

Fig. 2. Temperature dependence of the spin-lattice relaxation rate $1 / T_{1}$ of $\mathrm{Cu}^{2+}$ in triglycine sulphate crystal $(\bullet)$ and for $\mathrm{Cu}^{2+}$ and $\mathrm{Mn}^{2+}$ in $\left(\mathrm{NH}_{4}\right)_{2} \mathrm{Mg}\left(\mathrm{SO}_{4}\right)_{2} \cdot 6 \mathrm{H}_{2} \mathrm{O}$ crystal (triangles). The solid lines are the best fits obtained using the analytical approximations of $I_{n}$-values with parameters collected in Table II. 
TABLE II

Parameters of the relaxation equation $1 / T_{1}=a T+b T^{n+1} I_{n}\left(\Theta_{\mathrm{D}} / T\right)$.

\begin{tabular}{c|c|c|c|c|c}
\hline \hline Compound & $n$ & $\begin{array}{c}a \\
{\left[\mathrm{~K}^{-1} \mathrm{~s}^{-1}\right]}\end{array}$ & $\begin{array}{c}b \\
{\left[\mathrm{~K}^{-(n+1)} \mathrm{s}^{-1}\right]}\end{array}$ & $\begin{array}{c}\Theta_{\mathrm{D}} \\
{[\mathrm{K}]}\end{array}$ & Ref. no. \\
\hline triglycine sulphate (TGS): $\mathrm{Cu}^{2+}$ & 8 & 96 & $7 \times 10^{-14}$ & 190 & this paper \\
$\left(\mathrm{NH}_{4}\right)_{2} \mathrm{Mg}\left(\mathrm{SO}_{4}\right)_{2} \cdot 6 \mathrm{H}_{2} \mathrm{O}: \mathrm{Cu}^{2+}$ & 8 & 1700 & $8 \times 10^{-13}$ & 238 & {$[22]$} \\
$\left(\mathrm{NH}_{4}\right)_{2} \mathrm{Mg}\left(\mathrm{SO}_{4}\right)_{2} \cdot 6 \mathrm{H}_{2} \mathrm{O}: \mathrm{Mn}^{2+}$ & 6 & 140 & $4 \times 10^{-14}$ & 241 & {$[21,22]$}
\end{tabular}

compared with the results for $\left(\mathrm{NH}_{4}\right)_{2} \mathrm{Mg}\left(\mathrm{SO}_{4}\right)_{2} \cdot 6 \mathrm{H}_{2} \mathrm{O}$ crystal doped with $\mathrm{Cu}^{2+}$ and $\mathrm{Mn}^{2+}$ ions. The solid lines in Fig. 2 are the best fits with Eq. (11) with parameters collected in Table II. The Debye temperature $\Theta_{\mathrm{D}}$ values obtained for two different ions in $\left(\mathrm{NH}_{4}\right)_{2} \mathrm{Mg}\left(\mathrm{SO}_{4}\right)_{2} \cdot 6 \mathrm{H}_{2} \mathrm{O}$ lattice are practically the same with $\Theta_{\mathrm{D}}=240( \pm 5) \mathrm{K}$ indicating a correctness of the data analysis of the good accuracy results. In TGS crystal the Raman two-phonon relaxation processes dominate up to $75 \mathrm{~K}$. For higher temperatures an additional relaxation mechanism becomes dominating which can be related to the optical-phonon contribution.

\section{References}

[1] W.E. Rogers, R.L. Powell, Natl. Bur. Stand. (US) Circ. 1, 1 (1958).

[2] Landolt-Börnstein, Physikalisch-Chemische Tabellen, 5th ed., Berlin 1927.

[3] J.M. Ziman, Proc. R. Soc. 226, 436 (1954).

[4] I.N. Kurkin, Yu.K. Tshirkin, V.I. Shlenkin, Fiz. Tverd. Tela 14, 2719 (1972).

[5] S.K. Hoffmann, W. Hilczer, J. Goslar, J. Magn. Reson. 122, 37 (1996).

[6] S.K. Hoffmann, W. Hilczer, J. Goslar, M.M. Massa, R. Calvo, J. Magn. Reson. 153, 92 (2001).

[7] Ch. Kittel, Introduction to Solid State Physics, 3rd ed., Wiley, New York 1977, Ch. 7 .

[8] J.S. Blakemore, Solid State Physics, 2nd ed., Cambridge Univ. Press, Cambridge 1985, Ch. 3.

[9] D.G. Cahill, R.O. Pohl, Ann. Rev. Phys. Chem. 39, 93 (1988).

[10] P.G. Klemens, in: Encyclopedia of Physics, Vol. XIV, Low Temperature Physics 1, Springer, Berlin 1956, p. 198.

[11] R. Pirc, B. Žeks, P. Gosar, J. Phys. Chem. Solids 27, 1219 (1966).

[12] J.A. Sussmann, J. Phys. Chem. Solids 28, 1643 (1967).

[13] F.S. Ham, in: Electron Paramagnetic Resonance, Ed. S. Geschwind, Plenum, New York 1972, Ch. 1.

[14] P.L. Scott, C.D. Jeffries, Phys. Rev. 127, 32 (1962).

[15] R. Orbach, in: Electron Paramagnetic Resonance, Ed. S. Geschwind, Plenum, New York 1972, Ch. 2.

[16] R. Orbach, M. Blume, Phys. Rev. Lett. 8, 478 (1962). 
[17] K.P. Lee, D. Walsh, Phys, Lett. A 27, 17 (1968).

[18] I.M. Zaritskii, V.Y. Bratus, V.S. Vikhnin, A.S. Vishnevskii, A.A. Kothits, V.M. Ustintsev, Fiz. Tverd. Tela 18, 3226 (1976).

[19] V.S. Vikhnin, Fiz. Tverd. Tela 20, 1340 (1978).

[20] S.A. Altshuler, B.M. Kozyrev, Electron Paramagnetic Resonance of Transition Group Elements, 2nd ed., Nauka, Moscow 1972, p. 234.

[21] S.K. Hoffmann, M.A. Augustyniak, J. Goslar, W. Hilczer, Mol. Phys. 95, 1265 (1998)

[22] S.K. Hoffmann, W. Hilczer, J. Goslar, M.A. Augustyniak-Jablokow, J. Phys., Condens. Matter 13, 7443 (2001). 